ACTA BOTANICA MALACITANA es una revista de carácter internacional y de periodicidad anual que tiene por objeto la transmisión de los resultados de trabajos originales relacionados con cualquier campo de la fitología y, especialmente, aquellos dedicados a la sistemática, corología y ecología, aplicados tanto a las disciplinas de la criptogamia como de la fanerogamia.

Accredited with the International Association for Plant Taxonomy for the purpose of registration of all new plant names.

ABM es editada por el Departamento de Biología Vegetal y publicada por el Servicio de Publicaciones e Intercambio Científico de la Universidad de Málaga, con la colaboración de la Dirección General de Universidades e Investigación de la Consejería de Educación y Ciencia de la Junta de Andalucía.

\title{
Comité editor
}

Editor en jefe: B. Cabezudo; Editora ejecutiva: M.M. Trigo; Editores asociados: A. Flores, A.V. Pérez Latorre y T. Navarro; Editora edición electrónica: M. Recio.

\section{Comité asesor}

G. Blanca, Granada; F.D. Calonge, Madrid; C. Casas, Barcelona; M. Costa, Valencia; A. Crespo, Madrid; J.A. Devesa, Córdoba; T.E. Díaz, Oviedo; E. Domínguez, Córdoba; C. Favarger, Neuchâtel; M. Fennane, Rabat; J. Guerra, Murcia; J.P. Hébrard, Marseille; C. Herrera, Sevilla; J. Izco, Santiago de Compostela; X. Llimona, Barcelona; G. López, Madrid; E. Nardi, Firenze; J.L. Pérez-Cirera, Santiago de Compostela; S. Rivas-Martínez, Madrid; C. Sáenz, Madrid; P. Sánchez, Granada; J.A. Seoane, Barcelona; S. Talavera, Sevilla; B. Valdés, Sevilla; J. Varo, Granada.

Han colaborado como asesores en este volumen: N. Andreaki, Nápoles; E. Dana, Almería; A. Galán, Madrid; G. Moreno, Madrid; S. Ortiz, Santiago de Compostela; J. El Oualidi, Rabat; E. Rico, Salamanca; C. Rodríguez-Prieto, Girona; C. Romero Zarco, Sevilla.

\section{Secciones fijas}

Notas pteridológicas. Coordinador: B. Cabezudo.

Fragmentos taxonómicos, corológicos, nomenclaturales y fitocenológicos. Coordinadores: T. Navarro y A.V. Pérez Latorre.

Notas históricas. Coordinador: A. González Bueno.

Fragmenta palynologica. Coordinadores: G. Blanca y M.M. Trigo.

Contribuciones a la flora vascular de Andalucía (España). Coordinadores: C. Morales y B. Cabezudo.

Contribuciones a la flora vascular de Marruecos. Coordinadores: M. Fennane y J. El Oualidi.

Novedades taxonómicas y sintaxonómicas. Coordinadores: T. Navarro y A.V. Pérez Latorre.

Notulae taxinomicae, chorologicae, nomenclaturales, bibliographicae aut philologicae in opus "Flora Iberica" intendentes. Coordinador: S. Castroviejo.

Publicada en Diciembre de 2008

Maquetación: $\mathrm{M}^{\mathrm{a}}$ Dolores Tirado

Administración: Eva Linero

DEPARTAMENTO DE BIOLOGÍA VEGETAL

FACULTAD DE CIENCIAS

UNIVERSIDAD DE MÁLAGA

Apdo. 59; Fax 9521319 44. Tel. 952133342

E-mail: abm@uma.es

29080, MÁLAGA (ESPAÑA)

http://www.uma.es/Estudios/Departamentos/BiolVeg/03Rev/00HRev/01Rev.html

I.S.S.N. 0210 - 9506. Depósito Legal: MA. 338 - 1979.

Impresa en Andalucía

SERVICIO DE PUBLICACIONES

E INTERCAMBIO CIENTÍFICO

UNIVERSIDAD DE MÁLAGA MÁLAGA (ESPAÑA) 